\section{Unmet needs in human genomic variant interpretation}

To the Editor: In her Invited Commentary, Heidi Rehmchair of the ClinGen group setting up and sourcing the ClinVar database at the National Center for Biotechnology Information-made a call to action in response to unmet needs in human genomic variation interpretation. ${ }^{1}$

We fully appreciate and support scientific and community efforts to further standardize and improve variant classification, such as adding a scoring system or demonstrating how efficient guideline implementation could reduce discordance between different institutions performing the "same" variant classification routines. In fact, the level of quality in ClinVar's database workflows, data curation, and validation of medical interpretation is not yet proven, and in some aspects biased or even wrong.

The quality and the precision of a final diagnosis do not consist solely of correct variant classification and clinical interpretation. Diagnosis begins at the preanalytical level, and all subsequent interpretation can produce either a negative or a positive effect, depending on the quality of the "wet-bench" preparation. The College of American Pathologists (CAP) along with the US Food and Drug Administration (FDA) have developed a checklist and other materials to address this variability in order to safeguard "clinical validity" with respect to patient safety, making it a top priority (CAP ChecklistMolecular Pathology; FDA Laboratory Developed Test draft guidance documents).

If we acknowledge that any testing laboratory must (i) generate a data set that includes the relevant genetic variant, (ii) select this variant from among others, and (iii) classify this variant for its validity in clinical decisions, it follows that a high-quality process should be consistently executed at each step of the workflow. We strongly disagree that submitting classified variants to ClinVar increases the accuracy per se. Instead, we believe that the diagnostic community has improved its data quality and "caught up" as a result of the successful harmonization, standardization, and crossvalidation of variant classification initiated by the American College of Medical Genetics and Genomics (ACMG) working group.

We understand that Dr Rehm has a high opinion of ClinVar as a global resource serving all communities to facilitate data exchange for classification of rare-disease variants. However, as with any such database, the maturation of a database to provide "knowledge" over "data" requires a rigid quality-management and curation process.
Despite its obvious ambition and current level of funding, the ClinVar working group has yet to address the following issues:

1. ClinVar and other data repositories have yet to prove they can cope with the volume of submitted data and the dissection of eligible and noneligible data sets or track the quality of contributions over the long term. A global reference database for classification of rare disease variants must apply standardized high-quality criteria to assess the eligibility of all data plus internal curation to ensure that correct clinical interpretation does not become a matter of consensus or concordance. Without such a system in place, the risk to future majority decisions (and it is not clear whether the "majority decision" drives quality in all processes), considering the existing flaws and biases, is considerable.

2. The proposed ClinVar "star"-oriented rating system for evidence is heavily "expert"-centric. The star rating system should include justification of the suitability of "experts" and fair and comprehensive representation, with sufficient numbers of people carrying out the rating to avoid delay due to lack of availability. The relative weight of expert scores compared to technical scores must also be carefully and transparently defined to ensure a validated and revisable decision.

3. Setup and maintenance of ClinVar require substantial public resources. We need a fair discussion on alternative commercial and noncommercial models for improvement of the clinical validity of genetic testing before action is taken. The history of the Human Gene Mutation Database taught us that noncommercial initiatives usually have an early honeymoon period whereas a commercial model must be discussed and implemented early enough to provide a sustainable model. The conflict of interest underlying Heidi Rehm's commentary, as indicated by the exclusion of any other high-quality database, particularly her "call to action," is of concern. The sustainability of ClinVar for the next 510 years and beyond is a fundamental issue that must be addressed in order to generate trust in its model.

4. Last, but not least, a patient must have the ability to decide where to release their information and who they want using it. Forcing data generators to submit to ClinVar jeopardizes this control and is inappropriate.

To conclude, instead of a monopoly situation with respect to funding (i.e., the NIH funding process forcing laboratories to submit to ClinVar instead of any other high-quality database), guidance (ACMG and the ClinGen working group with a visible interest in lobbying for ClinVar), and global representation (what about Asian and European initiatives to promote the clinical validity of test interpretation?), we would 


\section{LETTER TO THE EDITOR}

like to see a call to action that encourages an unbiased evaluation of the advantages and limitations of database concepts and tools available worldwide, fosters communication and harmonization, and highlights all activities where the value for patients is maximized.

\section{DISCLOSURE}

The authors are employed by Centogene AG, a private genetic testing company that offers genetic services and has developed a fully curated, proprietary mutation database, CentoMD (r). Centogene fully shares genetic information, including classified variants, with referring physicians and patients' families upon request, but does not contribute to the ClinVar database because of the concerns outlined here. Otherwise, the authors declare no conflict of interest.

Peter Bauer, $M D^{1}$, Ellen Karges, $M S^{1}$, Gabriela Oprea, $\mathrm{PhD}^{1}$ and Arndt Rolfs, $M D^{1}$

${ }^{1}$ Centogene AG, Rostock, Germany. Correspondence: Peter Bauer (peter.bauer@centogene.com)

\section{REFERENCES}

1. Rehm HL et al. A new era in the interpretation of human genomic variation. Genet Med 2017;19:1092-1095.

Advance online publication 26 October 2017. doi:10.1038/gim.2017.187 